Anuario da Facultade de Dereito da Universidade da Coruña

Vol. 23 (2019), pp. 492-500

ISSNe: 2530-6324 || ISSN: 1138-039X

DOI: https://doi.org/10.17979/afdudc.2019.23.0.6036

\title{
SOBRE LA OPERATIVIDAD Y RESULTADOS DE LA UTILIZACIÓN DE LAS NUEVAS TECNOLOGIAS EN LA ENSEÑANZA DEL DERECHO ROMANO: UNA EXPERIENCIA PERSONAL *
}

\section{ON THE OPERABILITY AND RESULTS OF THE NEW TECHNOLOGIES IN THE FIELD OF ROMAN LAW TEACHING: A PERSONAL EXPERIENCE}

\author{
RAMÓN P. RODRÍGUEZ MONTERO \\ Prof. Titular de Derecho Romano \\ Universidade da Coruña
}

Resumen: Reflexiones personales sobre la utilización de las nuevas tecnologías en la enseñanza del Derecho romano.

Abstract: Personal reflections on the use of the new technologies in the field of roman law teaching.

Palabras clave: nuevas tecnologías, enseñanza universitaria, Derecho romano, experiencia docente.

Keywords: new technologies, university teaching, Roman Law, teaching experience.

Desde nuestra experiencia personal, que se concreta temporalmente en la prestación de servicios de forma exclusiva e ininterrumpida durante treinta y tres años como profesor de la asignatura Derecho romano en la Universidad española, consideramos que probablemente son dos los que parecen presentarse como como los argumentos más sólidos que pueden servir , en cierta medida y de forma condicionada,

\footnotetext{
* El presente escrito se corresponde con la versión leída que fue aportada como comunicación a la Sección 2 (“Enseñanza y práctica del Derecho en el mundo de las nuevas tecnologías”) del X Congreso de la Conferencia permanente de las Academias Jurídicas de Iberoamérica, organizado por la Real Academia de Jurisprudencia y Legislación de España y celebrado en Madrid los días 22 a 24 de noviembre del año 2018. La versión escrita del mismo, con las correspondientes referencias bibliográficas y notas a pie de página, aparecerá publicada en el Libro conmemorativo del Congreso.
} 
para justificar el recurso a la utilización de las nuevas tecnologías en la enseñanza de la materia que impartimos:

- Por una parte, la carga lectiva asignada a la asignatura en los Planes de estudios por el nuevo Espacio Educativo Europeo Superior (EEES), que ha supuesto una considerable reducción de las horas de docencia al estilo tradicional, frente a la señalada en el antiguo y añorado Plan de 1953.

- Por otra, la implantación desde el año 2010 en las Universidades españolas del denominado sistema de "Evaluación continua".

La reducción de la carga lectiva asignada a la asignatura en los Planes de estudios desde la reforma de 1990 y en el nuevo EEES, con el consiguiente recorte de horas de docencia al estilo tradicional - perfectamente apreciable si se comparan con las asignadas a aquélla en el Plan de 1953 - desgraciadamente ha supuesto para los docentes, a consecuencia de la necesidad de adaptación al escaso tiempo que se nos ha asignado, una considerable reducción del contenido de los programas de nuestra materia.

La —más que necesaria, obligada - reducción del contenido de nuestra materia, que paulatinamente ha ido perdiendo peso específico en la formación de los alumnos de Derecho, no ha seguido, como hubiera sido deseable y se echa en falta, un criterio uniforme establecido, por lo menos en sus aspectos básicos, por parte de las autoridades oficiales educativas, ni, en su caso, consensuado por los propios profesores de la asignatura de las distintas Universidades españolas, siendo tan grandes las diferencias apreciables entre los Planes de estudios de Derecho vigentes en España como para poder afirmar que en ocasiones los mismos solo tienen en común la denominación de la titulación.

Así, en atención a la obligada reducción del contenido de la materia, a preguntas que necesariamente nos hemos tenido que formular, como, por ejemplo, ¿qué conocimientos consideramos que debemos transmitir para formar juristas?, ¿qué queremos que nuestros discentes sepan?, ¿qué tenemos que seleccionar a efectos de su explicación e incluir en el programa de la asignatura?, en definitiva, ¿qué debemos explicar?, se ha contestado de forma diferente, dependiendo las respuestas proporcionadas a las mismas por los profesores de la distinta importancia y valoración - en definitiva, preferencia particular - que se atribuyese a los contenidos que en su consideración de conjunto conforman la asignatura —incidiendo con mayor intensidad en algunos, en detrimento, o incluso en exclusión de otros no menos importantes-, lo que, con carácter general, ha dado lugar a que la formación romanística recibida por los alumnos resulte desigual en las distintas Facultades de Derecho de las Universidades españolas.

Precisamente, respecto a la trascendental cuestión relativa a los conocimientos que consideramos que se deben de transmitir, a nadie escapan las dificultades con que nos encontramos los docentes de asignaturas formativas que, como el Derecho romano, se encuentran situadas en el primer curso del Grado.

Dichas dificultades se concretan fundamentalmente en la circunstancia de que los alumnos que inician sus estudios en el Grado en Derecho, en su gran mayoría, por 
no decir en su totalidad, al ingresar en la Universidad, por su edad y falta de contacto, de experiencia o de mera curiosidad, desconocen las que se presentan como realidades jurídicas cotidianas básicas, su estructura, características definitorias y diferenciadoras con otras similares o parecidas, los efectos y problemática cotidiana que en torno a las mismas se pudiesen plantear, etc.

Con la finalidad de intentar, si no solventar, por lo menos paliar en alguna medida esas carencias iniciales y poder profundizar en el estudio y desarrollo de esas realidades o conceptos jurídicos en la mayor medida de lo posible, consideramos que necesariamente debemos de asumir y partir de ese completo desconocimiento de lo jurídico por nuestros alumnos, y comenzar ordenadamente las explicaciones por el "léxico jurídico", para continuar con la "sintaxis jurídica" —ambas proporcionadas, como es sabido, en su esencia por los romanos-, y poder obtener, de esta forma, una base que permita a los discentes "aprender a leer jurídicamente” y estar en disposición de "entender lo que jurídicamente han leído". Todo ello, como es de suponer, implica la inversión de un tiempo que debemos de restar al poco del que de por sí se nos ha asignado, pero la tarea indicada creemos que es imprescindible.

Pensando en ese tiempo que necesariamente debemos invertir con los alumnos recién ingresados en la Universidad, hemos tenido la oportunidad de sugerir en otro escrito la posibilidad de introducir contenidos jurídicos básicos, tanto a nivel teórico, como en su proyección práctica, en las Enseñanzas Medias, fundamentalmente a través de la asignatura optativa denominada "Cultura clásica”, o también mediante otra, específicamente jurídica, que fuese creada al efecto para todos aquellos alumnos —que no son pocos - que tuviesen intención de realizar estudios de Derecho, o que, simplemente, deseasen llevar a cabo por mera curiosidad intelectual una aproximación en sus aspectos más elementales al ámbito jurídico.

En nuestra opinión, ello podría ayudar a reducir notablemente el obligado tiempo a invertir con los alumnos recién ingresados en el Grado en Derecho para sentar las necesarias bases jurídicas de que partir a los efectos de poder comenzar desarrollando —desde el conocimiento y comprensión imprescindibles de esas bases, como es lógico— la exposición de aspectos más técnicos y complejos.

Sería quizá aquí, en las Enseñanza Medias —y quizá no solamente en éstas, sino también en las Enseñanzas Universitarias-, donde podrían encontrar un encaje, siempre, claro está, como medios de transmisión del conocimiento que se pretende comunicar a los alumnos y dirigidas a ayudar a una mejor, más rápida y completa comprensión del mismo, las nuevas tecnologías.

Como posible sugerencia planteamos, por ejemplo, la confección de documentales. Pensamos, eso sí, en auténticos documentales, es decir, en películas informativas de contenidos o temas estrictamente jurídicos. Y decimos esto, porque en internet, bajo el título de videos de Derecho romano podemos encontrar videos en los que simplemente aparecen filmados profesores exponiendo temas o cuestiones determinadas de la asignatura, o bien, se trata de exposiciones en las que se utilizan exclusivamente esquemas o presentaciones de power point.

En esos documentales, mediante las correspondientes imágenes —que, según se dice coloquialmente, valen más que mil palabras - se procedería a proporcionar 
información — desde la realidad práctica de la vida cotidiana y los problemas que en la misma se plantean - a los discentes sobre diferentes instituciones o figuras jurídicas que, en muchas ocasiones, cuesta comprender a los alumnos a partir de la lectura de los libros o de las explicaciones de viva voz proporcionadas por el profesor. Como posibles ejemplos de lo indicado, por experiencia propia, podemos señalar, entre otros muchos: el funcionamiento de la in iure cesio, el carácter causal de la traditio, la estructura, funcionamiento y efectos de las obligaciones naturales, las diferencias y efectos de las obligaciones surgidas mediante pactos o estipulaciones, el momento de perfeccionamiento de los distintos tipos de contratos, etc.

Para la realización de esos documentales consideramos que resultaría necesario recurrir a la ayuda de expertos en Comunicación audiovisual con la finalidad de que, partiendo de los guiones elaborados por los profesores de Derecho del contenido que quisiesen transmitir, aquellos procediesen a plasmar los mismos — con las correcciones y sugerencias que estimasen oportuno introducir en los mismos desde su experiencia comunicativa - en los dichos documentales explicativos, que se pondrían a disposición de los alumnos, con la posibilidad, tras ser visionados, de comentar y ampliar los aspectos tratados en los mismos.

Por lo que respecta al posible contenido a presentar de la asignatura en la Universidad, en nuestra opinión, éste debería procurar ser, dentro de las limitaciones temporales asignadas para su impartición, lo más completo posible.

Así, en el cronograma que venimos realizando cada curso y que proporcionamos al inicio del mismo a los alumnos, en las aproximadamente quince semanas de que disponemos dentro del primer cuatrimestre del primer curso — en que se encuentra encuadrada nuestra asignatura como troncal-obligatoria-, para la impartición de la materia —a la que se asignan 6 créditos ECTS - dedicamos las 5 primeras semanas a aspectos relativos al Derecho público romano, incluyendo una referencia a la tradición romanística en la cultura jurídica europea, y las 10 restantes al Derecho privado romano, intentando que los alumnos dispongan de una visión de conjunto, si bien no todo lo amplia que nos gustaría por razón del tiempo asignado, sí, por lo menos, lo más completa posible de la asignatura, procurando aprovechar al máximo los potenciales aspectos formativos que la misma presenta y que entendemos que puede ofrecer a los alumnos.

De esta forma, la que consideramos como imprescindible exposición de determinados aspectos básicos y configuradores del denominado Derecho público romano o, si se prefiere, de la Historia del Derecho romano, en su consideración como asignatura histórica, permiten al alumno, entre otras muchas cosas, adquirir una mentalidad abierta y dinámica en relación al Derecho, viendo cómo ha ido cambiando, por qué ha cambiado, y cómo puede influir un determinado sistema político sobre la configuración jurídica de que se trate.

Por lo que se refiere al concreto ámbito del Derecho privado, como es sabido, el Derecho romano aporta fundamentalmente la terminología, los conceptos jurídicos básicos, y la técnica jurisprudencial del razonamiento jurídico.

Estos dos aspectos básicos son los que creemos que se deben de tomar como referencia imprescindible en relación a la posible presentación del contenido 
correspondiente al Derecho privado de la asignatura. Así, en las 10 semanas asignadas a la indicada parte procuramos ofrecer a los alumnos la información terminológica básica y las características identificadoras y definitorias más resaltables, correspondientes a las diversas instituciones y figuras jurídicas integradas en cada una de esas partes, siempre desde una visión diacrónica, e incidiendo en sus aspectos más prácticos y problemáticos.

Dichas explicaciones básicas de las partes indicadas, las realizamos recurriendo a impartirlas presencialmente, de forma verbal en el aula, durante las 2 horas asignadas semanalmente a la asignatura, establecidas para el grupo de alumnos en su conjunto, completándolas, o, en su caso, desarrollándolas, mediante exposiciones de aspectos concretos relativos a las mismas, en las 3 horas restantes semanales asignadas a cada uno de los 3 subgrupos en que se divide el grupo total de alumnos (correspondiendo 1 hora a cada 1 de los 3 subgrupos).

Estas últimas exposiciones son realizadas voluntariamente en cada uno de los subgrupos por los alumnos integrantes de los mismos de forma individual o colectiva, siempre bajo la supervisión del profesor, aprovechando las mismas para contrastar su nivel de conocimientos y comprensión de la materia expuesta, además de para, en su caso, proceder a aclarar las posibles dudas que les pudiesen surgir en lo expuesto.

Para llevar a cabo dichas exposiciones - para las que deben obtener la información correspondiente, guiándoseles y ayudándoseles por el profesor en los criterios de búsqueda-, los alumnos pueden utilizar, también voluntariamente, el recurso al power-point.

En relación a la utilización de este recurso tecnológico, a lo largo de estos años hemos venido observando que, llamativamente, en reiteradas ocasiones, muchos de los alumnos que se decantan por su utilización, siendo brillantes las presentaciones y exposiciones que realizan de los temas asignados, su nivel de comprensión de los contenidos expuestos, tras preguntarles por los mismos, adolece en bastantes casos de carencias importantes, precisamente por no haber comprendido bien, o en su caso de forma incorrecta, los que tan brillantemente han expuesto. A ello cabe añadir que, también en muchas otras ocasiones, los que utilizan en sus exposiciones el power-point, se limitan durante las mismas, simplemente, a dar lectura de lo contenido en cada imagen proyectada, haciéndose dichas presentaciones notablemente anodinas y desvirtuando la función auxiliar, de ayuda y clarificadora que, en relación a la exposición y explicación de los contenidos que se pretenden exponer, supuestamente ofrece dicho recurso.

Con la finalidad de evitar estos efectos no deseados y obtener el máximo partido del recurso tecnológico señalado, quizá resultase necesario organizar algunas actividades para los discentes en las que interviniesen de forma conjunta y coordinada tanto docentes de Derecho, para dar indicaciones a aquellos en cuanto al contenido a explicar y desarrollar en esas exposiciones — señalando al respecto, por ejemplo, qué es lo que se pretende exponer; qué es lo fundamental; en qué hay que incidir más y qué es lo accesorio, o que presenta menor importancia; cuál debe de ser el orden de exposición; etc.- , como docentes de Ciencias de la Comunicación, encargándose éstos de las cuestiones formales relativas a la exposición, es decir, de la manera más adecuada de realizarla con la finalidad de poder comunicar lo mejor posible y de la forma más 
efectiva, obteniendo así el nivel máximo de aprovechamiento del mencionado recurso interactivo.

Como complemento imprescindible de ayuda para la comprensión y resolución de las posibles dudas que pudiesen surgir a los alumnos del Grado en Derecho sobre la materia explicada por el profesor en las clases presenciales y la desarrollada voluntariamente por los alumnos en las exposiciones correspondientes a que hemos aludido anteriormente, las tutorías presentan una gran importancia.

A las tradicionales tutorías presenciales de un tiempo a esta parte se han añadido las "virtuales", impartidas a través del correo electrónico o bien mediante los foros habilitados al efecto a través de las respectivas plataformas por las correspondientes Universidades.

Sin perjuicio de manifestar nuestra preferencia por las tradicionales tutorías presenciales, al considerar necesario e insustituible el contacto personal entre el docente y el discente, entendemos que el correo electrónico, por el que en muchas ocasiones se suelen decantar los alumnos para contactar con el profesor, antes que hacerlo presencialmente, ofrece notables ventajas, especialmente para los que necesitan un asesoramiento, por así decirlo, urgente.

No obstante, a partir de nuestra experiencia, consideramos que los alumnos no han adquirido conciencia de la importancia del recurso a las tutorías, tanto presenciales como “virtuales”. Y decimos esto porque son más bien pocos los que, esporádicamente, recurren a las mismas a lo largo de los diferentes cursos académicos. Además, los que utilizan el correo electrónico lo suelen hacer para solicitar información —que generalmente ya se les ha proporcionado y de la que ya disponen- sobre el tipo de examen a realizar, número y forma de preguntas y materia objeto de evaluación, o, en su caso y en menor medida para preguntar sobre cuestiones de fondo relativas a los contenidos del programa de los que se tienen que examinar (formulando en ocasiones preguntas excesivamente amplias, que para ser contestadas adecuadamente requieren respuestas y matizaciones extensas) en fechas muy cercanas a las establecidas para realizar esas pruebas.

Respecto a las Plataformas digitales, que también se pueden utilizar como posibles recursos para hacer llegar información adicional de la materia (esquemas, preguntas tipo test, planteamiento de casos práctico, etc.) a los alumnos, e incluir posibles foros de debate, entendemos que deben resultar lo más claras y sencillas posibles en cuanto a su accesibilidad, estructura y manejabilidad, tanto para los discentes como para los docentes, lo que no siempre ocurre. De las que conocemos y hemos tenido oportunidad de utilizar, la que nos parece más apropiada es la denominada AKADEMOS, correspondiente a la UNED, que consideramos mucho más sencilla en cuanto a su uso y manejabilidad que la denominada MOODLE, utilizada en nuestra Universidad de La Coruña.

Sin renunciar a hacer uso de estos recursos tecnológicos cuando se estime oportuno, consideramos que, por lo que a la Universidad presencial se refiere, nunca se debe renunciar o minimizar el que entendemos como insustituible contacto personal entre el docente y el discente. También creemos que no está de más intentar “atenuar” la que a veces es excesiva dependencia de los medios tecnológicos. En este sentido, 
sugerimos a nuestros alumnos la realización voluntaria de resúmenes de partes concretas o cuestiones relativas a los contenidos de la asignatura, que necesariamente deben redactar a mano, evitando utilizar el habitual recurso al ordenador para ello.

Afortunadamente, en nuestra Universidad, además de disponer de la asignatura troncal obligatoria indicada, también se recoge en el Plan de Estudios otra optativa, denominada “Técnica jurisprudencial romana”, a la que se atribuyen 4,5 créditos y que se sitúa en el cuarto curso del Grado en Derecho.

El planteamiento docente que realizamos los profesores encargados de la misma, como se puede suponer, tiene un carácter mucho más práctico y técnico que el establecido para la asignatura troncal.

Contando con el conocimiento previo y comprensión de una serie de conocimientos romanísticos ya asimilados por los alumnos matriculados en la misma ya impartidos en la asignatura troncal-, se les propone o bien que realicen un caso práctico, o, en su caso, que analicen una determinada institución, figura, o cuestión jurídica de derecho privado, desde su vertiente doctrinal y jurisprudencial, en sus aspectos romanos, así como en su desarrollo histórico operado hasta el derecho vigente, debiendo exponer oralmente ante sus demás compañeros y los profesores de la asignatura los resultados del trabajo realizado. Para la realización de dichos trabajos, tras indicárseles la estructura formal que deben seguir, así como las cuestiones en que deben incidir, se les sugiere que, aparte de determinada información básica que se les proporciona, complementen la misma con el recurso adicional a la búsqueda, además de la contenida en escritos recogidos en papel, con los incluidos en internet y bases de datos informáticas, tanto textuales como doctrinales y jurisprudenciales. Respecto a la información contenida en internet, cabe indicar que, en algunos casos, la misma adolece de calidad suficiente por falta del necesario rigor científico o por su inexactitud, y se hace necesario, tras contrastarla, advertir a los alumnos que deben desecharla o matizar sus contenidos, planteamientos o conclusiones. Es evidente que, en no pocas ocasiones, el exceso de información ofrecido en la red, por resultar equívoca o distorsionadora para los alumnos, más que ofrecer ventajas para estos, puede resultarles claramente perjudicial. Otra de las posibles dificultades que se presentan en cuanto al manejo de dicha información incluida en internet radica en que la misma se recoge en idiomas distintos del nacional —en nuestro caso, el español-, cuyo desconocimiento por parte de los alumnos, desgraciadamente, les impide tener acceso a sus contenidos, que a veces resultan especialmente interesantes e instructivos.

En conclusión, sin negar la importancia de la utilización de las nuevas tecnologías en la enseñanza del Derecho tras la implantación del sistema ECTS y adaptación al EEES, consideramos que la misma hay que entenderla en sus justos términos. En este sentido, como se ha señalado muy sensatamente, esas nuevas tecnologías - seleccionando las que mejores resultados produzcan en el aprendizaje del discente- habrá que utilizarlas — siempre con sentido y sin excluir los recursos docentes tradicionales - como meros auxiliares didácticos, puesto que su función se limita a la trasmisión de información, dado que por sí mismas no crean conocimiento. Actúan, en definitiva —si se nos permite la expresión—, como "meros intermediarios" entre el docente y el discente, a los que "ponen en contacto y ayudan a comunicarse", lo que, en ocasiones, los que son fervientes defensores de su utilización parecen olvidar, exagerando la presunta excelencia de las mismas, planteándolos en algunos casos 
extremos con carácter exclusivo y excluyente frente a los métodos docentes tradicionales.

En nuestra opinión, no se puede olvidar — sin prescindir del recurso a las nuevas tecnologías, entendidas en los términos indicados- que es necesario e insustituible, en nuestro caso en las enseñanzas jurídicas, el contacto personal entre el docente y el discente, que indudablemente proporcionan con mayor intensidad y cercanía los recursos docentes tradicionales en las Universidades presenciales.

También se insiste en la circunstancia de que "la implantación del sistema ECTS, junto con la adaptación al EEES, conllevan un abandono de la docencia basada en la enseñanza del profesor, para acoger una docencia centrada en el aprendizaje del alumno; es decir, el profesor debe enseñar a aprender, y deberá ser el alumno el que asuma el protagonismo de su aprendizaje”.

Como también indicamos en otro escrito, consideramos que el objetivo descrito que se nos exige alcanzar no nos corresponde de forma exclusiva y excluyente a los profesores universitarios, siendo asimismo los profesores de las Enseñanzas Medias, previas a las universitarias, también en gran medida responsables y tributarios de su consecución. Por ello, sugerimos al efecto la que consideramos como deseable coordinación entre los docentes de dichos ámbitos educativos —Enseñanza Superior y Media - para poder alcanzarlo.

Es evidente que si los alumnos que, procedentes de las Enseñanzas medias, ingresan en la Universidad, lo hacen sin ser conscientes de ese papel protagonista en el aprendizaje que, de un tiempo a esta parte, se les viene atribuyendo —como generalmente observamos que viene ocurriendo-, adoptando el papel de meros receptores pasivos de conocimientos proporcionados por el profesor universitario (lo que tradicionalmente se viene calificando como “docencia basada en la enseñanza”), el esfuerzo de dichos profesores universitarios, como es lógico, se verá notablemente incrementado. $\mathrm{Y}$ esto es algo constatable, curso tras curso y salvo en contadas excepciones: con carácter general, los alumnos que recibimos de las Enseñanzas Medias -supuestos “protagonistas" de su aprendizaje universitario- se integran como meros elementos pasivos, cuyo único objetivo primordial se concreta en aprobar la asignatura de que se trate, a poder ser, con el menor esfuerzo posible.

$\mathrm{Si}$, por el contrario, esos alumnos llegasen debidamente concienciados desde sus estudios previos a la Universidad de su papel activo y protagonista en el aprendizaje, como teóricamente debería ocurrir, según se pretende, nuestra tarea -la de los profesores universitarios- se simplificaría notablemente.

Se dice que ahora el profesor debe “enseñar al alumno a aprender”, pero, sin embargo, no parece insistirse en algo tan simple e importante como que, para conseguirlo, el alumno — que es, recordamos, el que tiene que "asumir el protagonismo de su aprendizaje" - debe querer aprender, y que para ello debe tener interés, motivación, predisposición al aprendizaje, lo que en escasas ocasiones ocurre. Con carácter general, los alumnos que recibimos de las Enseñanzas Medias — supuestos "protagonistas" de su aprendizaje universitario - se integran - y ésta es una constante apreciable curso tras curso, salvo en contadas excepciones- como meros elementos 
pasivos, cuyo único objetivo primordial se concreta en aprobar la asignatura de que se trate con el menor esfuerzo posible.

Lo indicado en relación a la predisposición de los alumnos para asumir el idílico "papel protagonista" que se les atribuye en el nuevo sistema educativo nos vuelve a exigir a los docentes universitarios un nuevo esfuerzo, que no nos resulta desconocido por considerar que es imprescindible realizar desde siempre, y que se concreta en la necesidad de intentar justificar y convencer a nuestros alumnos de la supuesta utilidad del estudio y aprovechamiento de los conocimientos impartidos en nuestra asignatura, con la finalidad de conseguir motivarles para su estudio. Sin embargo, a nuestro juicio, no se incide, o, en su caso, se hace de forma insuficiente, en dos criterios que consideramos que se presentan como básicos y de los que hay que partir necesariamente para intentar obtener esos resultados que se pretenden, que son, además de la motivación e interés por aprender, el esfuerzo perseverante y la asunción de la consciencia del inconmensurable valor de la educación y la cultura. 